\title{
Vaspin, neutrophil gelatinase-associated lipocalin and apolipoprotein levels in patients with psoriatic arthritis
}

\author{
Colak $\mathrm{S}^{1}$, Omma A ${ }^{1}$, Sandikci $\mathrm{SC}^{1}$, Yucel $\mathrm{C}^{2}$, Omma $\mathrm{T}^{3}$, Turhan $\mathrm{T}^{2}$ \\ Ankara Gulhane Training and Research Hospital, Department of Rheumatology, Etlik Quarter, \\ Kecioren, Ankara, Turkey. sedayurumez@hotmail.com
}

\begin{abstract}
OBJECTIVES: To examine the relationship between disease activity and vaspin, neutrophil gelatinase-associated lipocalin (NGAL) and apolipoprotein levels in patients with psoriatic arthritis (PSA).

BACKGROUND: Psoriatic arthritis (PSA) is a chronic inflammatory arthritis, which is related with psoriasis. Adipokines are the mediators which play a role in metabolic homeostasis and inflammatory conditions.

METHODS: The levels of vaspin, NGAL, apolipoproteins and their correlations with disease activity were compared in 50 psoriatic arthritis patients and 36 healthy controls.

RESULTS: The levels of vaspin, NGAL and apolipoprotein B/A1 ratio were significantly higher in the patient group $(p<0.05)$. Apolipoprotein A1 was lower in the patients with PSA compared to the healthy control group. There was no significant correlation between disease activity and adipokines or metabolic parameters $(p>0.05)$. CONCLUSION: This is the first study to have compared vaspin and NGAL levels in patients with PsA. Vaspin and NGAL can be used as a biomarker in PsA. Vaspin, NGAL and dyslipoproteinemia are not correlated with disease activity (Tab. 3, Ref. 63). Text in PDF www.elis.sk.

KEY WORDS: apolipoprotein, disease activity, neutrophil gelatinase-associated lipocalin, psoriatic arthritis, vaspin.
\end{abstract}

\section{Introduction}

Psoriatic arthritis (PsA) is a chronic, inflammatory and debilitating arthritis associated with the skin disease, psoriasis (1). The incidence of psoriatic arthritis is $2-3 \%$ in the general population and $30 \%$ among patients with psoriasis (2). Although the etiology of PsA is not fully known, some risk factors have been identified. It has been shown that psoriatic arthritis patients are at increased risk for metabolic syndrome, obesity, cardiovascular events (CVEs), type 2 diabetes mellitus, hypertension and hyperlipidemia (3-6). Adipose tissue has an important role in the endocrine and immune systems by secreting adipokines. Some of these adipokines are anti-inflammatory, whereas some are pro-inflammatory (7).

Vaspin (visceral adipose tissue-derived serine protease inhibitor), a serine protease inhibitor, is a member of the adipokine group. Vaspin expression is increased in obesity. High vaspin levels are associated with increased glucose tolerance and insulin sensitivity and increased levels of vaspin are thought to play a protective role against the effects of insulin resistance (8). Vaspin can be the redeeming particle of metabolic syndrome pathogenesis (9). In recent trials, low vaspin levels have been found in cardiovascular

${ }^{1}$ Ankara Numune Training and Research Hospital, Department of Rheumatology, Ankara, ${ }^{2}$ Ankara Numune Training and Research Hospital, Department of Biochemistry, Ankara, and ${ }^{3}$ Ankara Training and Research Hospital, Department of Endocrinology and Metabolic Disorders, Ankara

Address for correspondence: Seda Colak, Ankara Gulhane Training and Research Hospital, Department of Rheumatology, Etlik Quarter, General Doctor Tevfik Saglam Street, No:1, Kecioren, Ankara, Turkey.

Phone: +905066268059 diseases $(10,11)$. It has been shown that vaspin protects endothelial cells from the effects of free fatty acids and prevents these cells from undergoing apoptosis, thereby playing a protective role against atherosclerosis (12).

Neutrophil gelatinase-associated lipocalin (Lipocalin-2, NGAL) is an immunomodulatory glycoprotein secreted by various cells, such as neutrophils, macrophages, adipocytes and hepatocytes. NGAL is a modulator of inflammatory response $(20,21,22)$. NGAL levels have been found to be high in inflammation, metabolic syndrome, cardiovascular diseases, acute kidney injury, lupus nephritis and malignancies (13-17).

The glycoprotein part of lipoproteins, which are the structures that allow the lipids to move in the blood and dissolve in water, are called apolipoproteins. The main apolipoproteins of high-density lipoproteins (HDL) and low-density lipoproteins (LDL) are apolipoprotein A1 (apo A1) and apolipoprotein B (apo B), respectively. There is a negative correlation between serum apo A1 concentration and atherosclerosis. Apo B has been shown to be related with atherosclerosis $(18,19)$.

The aim of this study was to evaluate the relationship between vaspin, NGAL levels, lipoproteins and disease activity in PsA patients.

\section{Materials and methods}

\section{Patients and disease activity assessment}

Samples were obtained from 50 PsA patients and 36 healthy control subjects between November 2017 and February 2018. Patients with PsA were defined according to the Classification Criteria 
for Psoriatic Arthritis (CASPAR) (20). The study exclusion criteria were as follows: 1 ) age $<18$ years 2 ) concomitant autoimmune or autoinflammatory disease, 3 ) acute or chronic infection, 4) malignancy, 5) pregnancy or up to 6 months postpartum period. The demographic features and clinical characteristics were recorded. Disease severity was assessed with the Psoriasis Area Severity Index (PASI) for skin disease. Arthritis activity was measured using the disease activity in PsA(DAPSA), Bath Ankylosing Spondylitis Disease Activity Index (BASDAI), Bath Ankylosing Spondylitis Functional Index (BASFI), Visual Analogue Scale (VAS) and Minimal Disease Activity (MDA) (1, 21-23). Body mass index (BMI) was calculated and waist circumference (WC) was measured for each individual. The presence of metabolic syndrome was evaluated with the criteria of the National Cholesterol Education Program Adult Panel III (NCEP ATP III) (24).

The study protocol was approved by the Local Ethics Committee. The research protocol complies with the 2000 Declaration of Helsinki and written informed consent was obtained from all participants.

\section{Laboratory analysis}

Venous blood samples were collected from the participants after 12 hours of fasting and centrifuged at $1,500 \mathrm{~g}$ for 10 minutes. Separated sera were aliquoted into Eppendorf tubes and stored at $-80{ }^{\circ} \mathrm{C}$ until the time of analysis.

Serum levels of Vaspin and NGAL were detected with commercial ELISA (double antibody sandwich ELISA method) test kit (Elabscience Biotech Co. Ltd) according to the manufacturer's protocol. HDL, cholesterol and triglyceride measurements were made with the enzymatic colorimetric method, LDL was calculated according to the Friedwald Formula. Apo B, Apo A1 and C reactive protein (CRP) values were detected with the immunoturbidimetric method. Erythrocyte sedimentation rate (ESR) was studied with Westergreen method.

\section{Statistical analysis}

Statistical analysis was performed with the Statistical Package for Social Sciences (SPSS) program version 18.0 for Windows (SPSS Inc., Chicago, IL, USA). Conformity to normal distribution of the variables was investigated using visual and analytical methods. Normally distributed continuous values were expressed as mean \pm standard deviation (SD) and categorical variables as number $(n)$ and percentage (\%). Parameters which were not normally distributed were stated as median values with interquartile range (IQR). Continuous parameters were analyzed using the Student's ttest. Correlations were evaluated with the Pearson correlation coefficient. A value of $p<0.05$ was considered statistically significant.

\section{Results}

Evaluation was made on the data of 50 PsA patients and 36 healthy control subjects. The groups were similar in respect of age and gender. Among PsA patients, metabolic syndrome, diabetes mellitus, hypertension and hyperlipidemia were seen in 21 $(40 \%), 8(16 \%), 13(26 \%)$ and $2(4 \%)$ patients, respectively.
Tab. 1. Comparison of the demographic and clinical variables of the patient and control groups.

\begin{tabular}{lccc}
\hline & PsA & Healthy control & P \\
\hline Male/Female $^{\mathrm{a}}$ & $35 / 15(70 / 30)$ & $25 / 11(69.4 / 30.5)$ & 0.956 \\
\hline Age & $44.58 \pm 13.88$ & $43.08 \pm 11.04$ & 0.59 \\
\hline Disease duration (months) & & & \\
$\quad$ Psoriasis & $121.36 \pm 97.64$ & & \\
$\quad$ Psoriatic arthritis & $59.83 \pm 55.8$ & & \\
\hline Involvement types $^{\mathrm{a}}$ & & & \\
$\quad$ Axial & $14(28)$ & & \\
Peripheral & $45(90)$ & & \\
$\quad$ Dactylitis & $22(44)$ & & \\
$\quad$ Enthesitis & $9(18)$ & & \\
\hline ESR mm/h & $20.2 \pm 16.27$ & & \\
\hline CRP mg/dl & $9.28 \pm 12.11$ & & \\
\hline BMI $\left(\mathrm{kg} / \mathrm{m}^{2}\right)$ & $29.78 \pm 5.42$ & $26.31 \pm 4.86$ & $\mathbf{0 . 0 0 3}$ \\
\hline WC $(\mathrm{cm})$ & $98.86 \pm 13.68$ & $89.2 \pm 10.67$ & $\mathbf{0 . 0 0 1}$ \\
\hline PSA $\mathrm{pog}$
\end{tabular}

PsA: psoriatic arthritis, ESR: erythrocyte sedimentation rate, CRP: C reactive protein, BMI: body mass index, WC: waist circumference. For the variables with ${ }^{\text {a }}$, frequency (percent), without ${ }^{\text {a }}$, mean \pm standard deviation was used.

Tab. 2. Disease activity scores of patients.

\begin{tabular}{lcc}
\hline & Mean \pm Standard deviation & Median $($ Minimum-Maximum) \\
\hline DAPSA & $19.5 \pm 18.63$ & $11.65(0-78)$ \\
BASDAI & $2.68 \pm 2.77$ & $2.2(0-10)$ \\
BASFI & $1.87 \pm 2.35$ & $1(0-9.6)$ \\
PASI & $7.91 \pm 13.6$ & $1.5(0-62.6)$ \\
VAS & $5.28 \pm 3.23$ & $6(0-10)$ \\
\hline
\end{tabular}

DAPSA: Disease activity in psoritic arthritis, BASDAI: Bath Ankylosing Spondylitis Disease Activity Index, BASFI: Bath Ankylosing Spondylitis Functional Index, PASI: Psoriasis Area Severity Index, VAS: Visual analogue scale

Tab. 3. Comparison of the adipokine and apolipoprotein levels of the patient and control groups.

\begin{tabular}{lccc}
\hline Variable & Patients & Controls & $\mathrm{p}$ \\
\hline Vaspin (ng/L) & $391.63 \pm 436.4$ & $176.67 \pm 122.75$ & $\mathbf{0 . 0 0 1}$ \\
NGAL (ng/L) & $5.2 \pm 2.67$ & $1.94 \pm 2.09$ & $\mathbf{0 . 0 1 4}$ \\
Apolipoprotein A1 (mg/dl) & $131.9 \pm 17.38$ & $141.72 \pm 19.97$ & $\mathbf{0 . 0 1 7}$ \\
Apolipoprotein B (mg/dl) & $102.52 \pm 27.29$ & $92.55 \pm 34.21$ & 0.137 \\
Apolipoprotein B/A1 & $0.78 \pm 0.21$ & $0.66 \pm 0.27$ & $\mathbf{0 . 0 2 3}$ \\
\hline
\end{tabular}

NGAL: Neutrophil Gelatinase-associated Lipocalin

BMI and WC were significantly greater in the patient group. Of PsA patients, 21 (24.4\%) had metabolic syndrome. Demographic and clinical variables are shown in Table 1 . The disease activity scores of patients are shown in Table 2. According to MDA criteria, $26(52 \%)$ patients were in remission.

Medications and the percentage of usage were analyzed. Twenty-one patients (42\%) were using corticosteroids, 31 patients (62\%) were using nonsteroidal anti-inflammatory drugs, 28 patients $(56 \%)$ were using methotrexate, 7 patients $(14 \%)$ were using salazopyrine, 5 patients ( $10 \%$ ) were using leflunomide, 1 patient (2\%) was using cyclosporine and 9 patients (18\%) were using anti tumour necrosis factor $\alpha$ blockers.

Vaspin and NGAL levels were higher in patients than in the healthy control group. The level of Apo A1 was lower and the Apo $\mathrm{B} / \mathrm{A} 1$ ratio was higher in the patient group $(\mathrm{p}=0.017 \mathrm{vs} \mathrm{p}=$ 0.023 , respectively) (Tab. 3). Patients with metabolic syndrome had higher NGAL, Apo B and Apo B/A1 ratio $(\mathrm{p}<0.05)$. 
There was no statistically significant correlation between disease activity and vaspin, NGAL, and lipoprotein levels ( $p>0.05)$.

NGAL levels had a negative correlation with disease duration of psoriasis and psoriatic arthritis $(\mathrm{p}<0.05)$. There was no correlation between BMI and waist circumference and levels of vaspin, NGAL, and Apo A1 ( $p>0.05)$. There was a positive correlation between Apo B, Apo B/A1 and BMI and WC ( $<<0.05)$. Patients with metabolic syndrome had higher scores of DAPSA and PASI $(\mathrm{p}<0.05)$.

\section{Discussion}

This study analyzed adipokines, apolipoproteins A and B, and their correlation with disease activity in patients with PsA. This is the first study to have assessed vaspin and NGAL levels and the correlations with disease activity in PsA.

The results of the study showed that BMI was higher in PsA patients, which was consistent with previous findings in literature (25-27). A recent study demonstrated that WC was higher in PsA patients which is similar to the current study findings (28). It is well known that metabolic syndrome is a common disorder among PsA patients $(3,4)$. In this study, metabolic syndrome was seen in 21 patients $(24.4 \%)$, which was a lower rate than previously reported in literature $(29,30)$.

Vaspin and NGAL levels were determined to be higher in PsA patients compared with the healthy control group. Vaspin plays a role in atherosclerosis, impaired insulin resistance, obesity and chronic inflammation $(31,32)$. Vaspin is known to have anti-inflammatory properties (33-35). The results of this study showed vaspin to be higher in the PsA patient group, but no correlation was determined between disease activity and vaspin levels. Maijer et al found that serum vaspin levels were associated with the development of arthritis in autoantibody positive individuals. In that study, vaspin levels were not associated with CRP (36). Metabolic syndrome, CVEs, and obesity are conditions known to change vaspin levels $(8,37,38)$, with lower levels seen in type 2 diabetes mellitus, endometrial and colon cancer patients (32, $39,40)$. The results of studies on vaspin levels in patients with psoriasis are contradictory. Coban et al showed that vaspin levels were higher in psoriasis patients than in the healthy control group, but no correlation was shown between vaspin levels and PASI scores (7). In another trial, the healthy control group vaspin levels did not differ from those of psoriasis patients (41). Ataseven et al showed that vaspin levels were lower in psoriasis compared with healthy control subjects and there was a positive correlation with PASI (42). Ozgen et al reported higher vaspin levels in RA patients, but there was no significant correlation between vaspin levels and disease activity. In the same study, vaspin levels were lower in active Behçet's disease (43). In a study of juvenile idiopathic arthritis patients, vaspin levels did not differ from those of the control group and no significant correlation was found between vaspin levels and disease activity (44). Wang et al demonstrated low vaspin levels in ankylosing spondylitis patients (45). Various chronic inflammatory diseases present different vaspin responses. There is a need for further studies to be able to better understand the role of vaspin in chronic inflammatory diseases. In the current study, no correlation was found between vaspin and BMI or WC, which was similar to the findings in literature (36).

NGAL, a member of the adipokine superfamily, is secreted by neutrophils, macrophages, adipocytes and epithelial cells. This molecule plays a role in the regulation of inflammation, infections, immunity, ischaemia-induced injury and metabolic balance of the human body $(46,47)$. It is known that IL-17 is one of the major cytokines of PsA. Shen et al demonstrated that IL-17 induces NGAL secretion (48). Secretion of NGAL can be regulated either IL-17 or the interaction between IL-17 and TNF- $\alpha$ (46). Although NGAL can be secreted from adipocytes, no correlation was found in the current study between NGAL and BMI or WC. However, there was a positive correlation between the presence of metabolic syndrome and levels of NGAL. To the best of our knowledge there is no study which has examined NGAL in psoriatic arthritis patients with which the current study results could be compared. Serum and tissue NGAL levels have previously been found to be increased in patients with psoriasis $(49,50)$. NGAL was not correlated with PASI, BMI or WC in studies which were conducted in patients with psoriasis (49-51). Kamata and Stejkal did not find a significant correlation between metabolic syndrome or its components and levels of NGAL in patients with psoriasis (50, 51). Romani et al showed a positive correlation between NGAL and PASI (52). Gulkesen et al reported that NGAL levels were higher in patients with RA than in the healthy control group and there was no correlation with disease activity (53).

In the current study, NGAL levels were negatively correlated with both psoriasis and psoriatic arthritis disease duration. In contrast, El Hadidi et al found no correlation between disease duration of psoriasis and NGAL levels (49).

Apolipoproteins provide transportation of lipids. Apo A1 is the major protein of HDL, which activates the enzyme, lecithin cholesterol acyltransferase (LCAT). This enzyme esterifies free cholesterol and removes cholesterol from peripheral cells. Apo A1 has antiatherogenic properties and prevents cross-interaction between macrophages and T cells. Changes in the protein and lipid structure of HDL occur in the presence of inflammation. Serum amyloid A is replaced by Apo A1 in HDL. Inflammation causes depletion of Apo A1. Apo B is the major protein of LDL, which provides transport of cholesterol from the liver to the periphery $(18,19,54,55)$. Although the exact value of the Apo B/A1 ratio is not fully known in literature, this ratio is considered to be a better predictor of cardiovascular risk than lipid and lipoprotein levels (56). In the current study, Apo A1 levels were found to be significantly lower and the Apo B/A1 ratio was significantly higher in the patient group. There was no significant correlation between apolipoprotein levels and disease activity. Apolipoprotein levels have been studied in various inflammatory diseases. Terkeltaub showed that Apo A1 levels were decreased in RA and increased in PsA patients (57). Gentile et al reported that Apo B levels were increased in patients with PsA(58), whereas Papagoras et al showed significantly lower Apo A1 levels in PsA patients and there were no significant differences in Apo B levels and ratio of Apo B/A1. There were no correlations between metabolic parameters and 
PASI, BASDAI, or DAS28. In the same study AS patients had lower Apo B and Apo B/A1 ratio. (59). Tam et al evaluated 31 PsA patients and reported higher Apo A1, Apo B and the ratio in PsA patients. According to that study, CRP had a negative correlation with Apo A1 and HDL, and a positive correlation with Apo $\mathrm{B} / \mathrm{A} 1$ ratio (27). Semb and Jones showed that lipid subfractions do not change in PsA $(60,61)$. Another study demonstrated that PsA patients had higher Apo B and LDL levels, and conversely lower Apo A1 and HDL levels compared with the healthy control group (62). Similar findings were reported in another study of psoriasis patients (63). To be certain of the role of apolipoproteins in PsA, there is a need for further studies with larger samples. The contradictory results of the previous studies could be due to demographic characteristics, pathophysiology of diseases or risk of cardiovascular events.

The limitation to the current study was in the small sample size of the patient group. The cross-sectional design of the study and the fact that there was no follow-up of the patients can also be considered as limitations.

\section{Conclusion}

Vaspin and NGAL can be used as positive markers in patients with PsA. These adipokines can play a role in the inflammatory process of PsA. Apolipoprotein levels can be showing the increased risk for CVEs in patients with PsA. The contradictory results in literature indicate the need for future studies with larger samples.

\section{Learning points}

- High vaspin levels were found in patients with psoriatic arthritis.

- Neutrophil geletinase-associated lipocalin was higher in psoriatic arthritis.

- Apolipoprotein A1 level is lower in the patient group.

- The ratio of apolipoprotein $\mathrm{B} / \mathrm{A} 1$ is higher among the psoriatic arthritis patients.

- There was no correlation between disease activity and these biomarkers.

\section{References}

1. Ritchlin CT, Colbert RA, Gladman DD. Psoriatic Arthritis. N Engl J Med 2017; 376: 957-970

2. Gladman DD. Recent advances in understanding and managing psoriatic arthritis. F1000Research 2016 Nov 16; 5: 2670. Available from: https: //f1000research.com/articles/5-2670/v1

3. Raychaudhuri SK, Chatterjee S, Nguyen C et al. Increased Prevalence of the Metabolic Syndrome in Patients with Psoriatic Arthritis. Metab Syndr Relat Disord 2010; 8: 331-334

4. Lin YC, Dalal D, Churton S et al. Relationship Between Metabolic Syndrome and Carotid Intima-Media Thickness: Cross-Sectional Comparison Between Psoriasis and Psoriatic Arthritis. Arthritis Care Res (Hoboken) 2014; 66: 97-103.

5. Horreau C, Pouplard C, Brenaut E et al. Cardiovascular morbidity and mortality in psoriasis and psoriatic arthritis: A systematic literature review. J Eur Acad Dermatology Venereol 2013; 27: 12-29.
6. Daïen CI, Sellam J. Obesity and inflammatory arthritis: impact on occurrence, disease characteristics and therapeutic response. RMD Open 2015 Jun 29; 1 (1): e000012. doi: 10.1136/rmdopen-2014-000012

7. Coban M, Tasli L, Turgut S et al. Association of adipokines, insulin resistance, hypertension and dyslipidemia in patients with psoriasis vulgaris. Ann Dermatol 2016; 28: 74-79.

8. Hida K, Wada J, Eguchi $\mathbf{J}$ et al. Visceral adipose tissue-derived serine protease inhibitor: a unique insulin-sensitizing adipocytokine in obesity. Proc Natl Acad Sci USA 2005; 102: 10610-10615.

9. Wada J. Vaspin: a novel serpin with insulin-sensitizing effects. Expert Opin Investig Drugs 2008; 17 (3): 327-333.

10. Kobat MA, Celik A, Balin M et al. The Investigation of Serum Vaspin Level in Atherosclerotic Coronary Artery Disease. J Clin Med Res 2012 April; 4: 110-113. doi: 10.4021/jocmr841w.

11. Blüher M. Vaspin in obesity and diabetes: Pathophysiological and clinical significance. Endocrine 2012; 41: 176-182.

12. Liu S, Dong Y, Wang $\mathbf{T}$ et al. Vaspin inhibited proinflammatory cytokine induced activation of nuclear factor-kappa B and its downstream molecules in human endothelial EA.hy926 cells. Diabetes Res Clin Pract 2014; 103: 482-488.

13. Shashidharamurthy $\mathbf{R}$, Machiah $\mathbf{D}$, Aitken $\mathbf{J}$ et al. Differential role of lipocalin-2 during immune-complex mediated acute and chronic inflammation. Arthritis Rheum 2013; 65: 1064-1073.

14. Ozemir IA, Aslan S, Eren T et al. The Diagnostic and Prognostic Significance of Serum Neutrophil Gelatinase-Associated Lipocalin Levels in Patients with Colorectal Cancer. Chirurgia (Bucur) 2016; 111: 414-421.

15. Ishii A, Katsuura G, Imamaki $\mathbf{H}$ et al. Obesity-promoting and antithermogenic effects of neutrophil gelatinase-associated lipocalin in mice. Sci Rep 2017 Nov 14; 7 (1): 15501. doi: 10.1038/s41598-017-15825-4.

16. Choi KM, Lee JS, Kim EJ et al. Implication of lipocalin-2 and visfatin levels in patients with coronary heart disease. Eur J Endocrinol 2008; 158: 203-207.

17. Rubinstein T, Pitashny M, Levine B et al. Urinary neutrophil gelatinase-associated lipocalin as a novel biomarker for disease activity in lupus nephritis. Rheumatology (Oxford) 2010; 49: 960-971.

18. Roheim PS, Asztalos BF. Clinical significance of lipoprotein size and risk for coronary atherosclerosis. Clin Chem 1995; 41: 147-152.

19. Ansell BJ, Watson KE, Fogelman AM, Navab M, Fonarow GC. Highdensity lipoprotein function: Recent advances. J Am Coll Cardiol 2005; 46: 1792-1798.

20. Taylor W, Gladman D, Helliwell P et al. Classification criteria for psoriatic arthritis: Development of new criteria from a large international study. Arthritis Rheum 2006; 54: 2665-2673.

21. Schoels MM, Aletaha D, Alasti F, Smolen JS. Disease activity in psoriatic arthritis (PsA): defining remission and treatment success using the DAPSA score. Ann Rheum Dis 2016; 75: 811-818.

22. Coates L. Outcome Measures in Psoriatic Arthritis. Rheum Dis Clin North Am 2015; 41: 699-710.

23. Coates L, Helliwell PS. Validation of minimal disease activity criteria for psoriatic arthritis using interventional trial data. Arthritis Care Res 2010; 62: 965-969.

24. Pehlevan S, Yetkin D, Bahadır C et al. Increased prevalence of metabolic syndrome in patients with psoriatic arthritis. Metab Syndr Relat Disord $2014 ; 12: 43-48$.

25. Kimhi O, Caspi D, Bornstein NM et al. Prevalence and Risk Factors of Atherosclerosis in Patients with Psoriatic Arthritis. Semin Arthritis Rheum 2007; 36: 203-209. 
26. Sharma A, Gopalakrishnan D, Kumar R et al. Metabolic syndrome in psoriatic arthritis patients: A cross-sectional study. Int J Rheum Dis 2013; 16: 667-673.

27. Tam L-S, Tomlinson B, Chu TT-W et al. Cardiovascular risk profile of patients with psoriatic arthritis compared to controls--the role of inflammation. Rheumatology 2008; 47: 718-723.

28. Ozkaya DB, Onsun N, Topukçu B et al. The relationship between body mass index, waist circumference and psoriatic arthritis in the Turkish population. Postep Dermatologii i Alergol 2016; 33: 219-223.

29. Ozkan SG, Yazisiz H, Behlul A et al. Prevalence of metabolic syndrome and degree of cardiovascular disease risk in patients with Psoriatic Arthritis. Eur J Rheumatol 2017; 4: 40-45.

30. Haroon M, Bin A, Chaudhry R, Fitzgerald O. Higher Prevalence of Metabolic Syndrome in Patients with Psoriatic Arthritis : A Comparison with a Control Group of Noninflammatory Rheumatologic Conditions. J Rheumatol 2016; 43: 3-5.

31. Pawar R, Goilav B, Xia Y et al. Serum Autoantibodies in Pristane Induced Lupus are Regulated by Neutrophil Gelatinase Associated Lipocalin Rahul. Clin Immunol 2014; 154: 49-65.

32. Sathyaseelan AJ, Adole PS, Wyawahare M, Saya RP. Assessment of Serum VASPIN Levels among Type 2 Diabetes Mellitus Patients with or without Acute Coronary Syndrome. J Clin Diagn Res 2016; 10 (12): BC07BC10. doi: 10.7860/JCDR/2016/22417.8952.

33. Saalbach A, Tremel J, Herbert D et al. Anti-inflammatory action of keratinocyte-derived vaspin relevance for the pathogenesis of psoriasis. Am J Pathol 2016; 186: 639-651.

34. Phalitakul S, Okada M, Hara Y, Yamawaki H. Vaspin prevents TNF$\alpha$-induced intracellular adhesion molecule-1 via inhibiting reactive oxygen species-dependent NF- $\kappa \mathrm{B}$ and PKC $\theta$ activation in cultured rat vascular smooth muscle cells. Pharmacol Res 2011; 64: 493-500.

35. Jung CH, Lee WJ, Hwang JY et al. Vaspin protects vascular endothelial cells against free fatty acid-induced apoptosis through a phosphatidylinositol 3-kinase/Akt pathway. Biochem Biophys Res Commun 2011; 413: 264-269.

36. Maijer KI, Neumann E, Mãller-Ladner U et al. Serum vaspin levels are associated with the development of clinically manifest arthritis in autoantibody-positive individuals. PLoS One 2015; 10: 1-13.

37. Aust G, Richter O, Rohm S et al. Vaspin serum concentrations in patients with carotid stenosis. Atherosclerosis 2009; 204: 262-266.

38. Kadoglou NPE, Gkontopoulos A, Kapelouzou A et al. Serum levels of vaspin and visfatin in patients with coronary artery disease-Kozani study. Clin Chim Acta 2011; 412: 48-52.

39. Erdogan S, Sezer S, Baser $\mathbf{E}$ et al. Evaluating vaspin and adiponectin in postmenopausal women with endometrial cancer. Endocr Relat Cancer 2013; 20: 669-675.

40. Fazeli M, Dashti H, Akbarzadeh S et al. Circulating levels of novel adipocytokines in patients with colorectal cancer. Cytokine 2013; 62: 81-85.

41. Saalbach A, Vester K, Rall K et al. Vaspin - a link of obesity and psoriasis? Exp Dermatol 2012; 21: 309-312.

42. Ataseven A, Kesli R. Novel inflammatory markers in psoriasis vulgaris: vaspin, vascular adhesion protein-1 (VAP-1), and YKL-40. G Ital Dermatol Venereol 2016; 151: 244-250.

43. Ozgen M, Koca S, Dagli $\mathbf{N}$ et al. Serum adiponectin and vaspin levels in rheumatoid arthritis. Arch Med Res 2010; 41: 457-463.

44. Cantarini L, Simonini G, Fioravanti A et al. Circulating levels of the adipokines vaspin and omentin in patients with juvenile idiopathic arthritis, and relation to disease activity. Clin Exp Rheumatol 2011; 29: 1044-1048.
45. Wang HH, Wang QF. Low vaspin levels are related to endothelial dysfunction in patients with ankylosing spondylitis. Brazilian J Med Biol Res 2016; 49: 1-6.

46. Ferreira MC, Whibley N, Mamo AJ et al. Interleukin-17-induced protein lipocalin 2 is dispensable for immunity to oral candidiasis. Infect Immun 2014; 82: 1030-1035.

47. Abella V, Scotece M, Conde J et al. The potential of lipocalin-2/NGAL as biomarker for inflammatory and metabolic diseases. Biomarkers 2015; 20: $565-571$.

48. Shen F, Hu Z, Goswami J, Gaffen SL. Identification of common transcriptional regulatory elements in interleukin-17 target genes. J Biol Chem 2006; 281: 24138-24148.

49. El-Hadidi H, Samir N, Shaker OG, Otb S. Estimation of tissue and serum lipocalin-2 in psoriasis vulgaris and its relation to metabolic syndrome. Arch Dermatol Res 2014; 306: 239-245.

50. Kamata M, Tada Y, Tatsuta A, K et al. Serum lipocalin-2 levels are increased in patients with psoriasis. Clin Exp Dermatol 2012; 37: 296-299.

51. Stejskal D, Karpísek M, Humenanska V et al. Lipocalin-2: development, analytical characterization, and clinical testing of a new ELISA. Horm Metab Res 2008; 40: 381-385.

52. Romaní J, Caixàs A, Ceperuelo-Mallafré V et al. Circulating levels of lipocalin-2 and retinol-binding protein-4 are increased in psoriatic patients and correlated with baseline PASI. Arch Dermatol Res 2013; 305: 105-112.

53. Gulkesen A, Akgol G, Poyraz AK et al. Lipocalin 2 as a clinical significance in rheumatoid arthritis. Cent J Immunol 2017; 42: 269-273.

54. Eder L, Harvey P. Cardiovascular Morbidity in Psoriatic Arthritis : What Is the Effect of Cardiovascular Morbidity in Psoriatic Arthritis: What Is the Effect of Inflammation? 2017; 44: 1295-1297.

55. Sniderman AD, Junger I, Holme I et al. Errors that result from using the TC/HDL C ratio rather than the apoB/apoA-I ratio to identify the lipoprotein-related risk of vascular disease. J Intern Med 2006; 259: 455-461.

56. Walldius G, Jungner I. The apoB/apoA-I ratio: A strong, new risk factor for cardiovascular disease and a target for lipid-lowering therapy - A review of the evidence. J Intern Med 2006; 259: 493-519.

57. Terkeltaub R. ApoA-I at the interface of vascular inflammation and arthritis. Arter Thromb Vasc Biol 2014; 34: 474-476.

58. Gentile M, Peluso R, Nicola $M$ et al. Association between small dense LDL and sub-clinical atherosclerosis in patients with psoriatic arthritis. Clin Rheumatol 2016; 35: 2023-2029.

59. Papagoras C, Markatseli TE, Saougou I et al. Cardiovascular risk profile in patients with spondyloarthritis. Jt Bone Spine 2014; 81: 57-63.

60. Semb AG, Kvien TK, DeMicco DA et al. Prediction of Cardiovascular Events in Patients with Ankylosing Spondylitis and Psoriatic Arthritis : Role of Lipoproteins in a High-risk Population J Rheumatol 2012; 39: 1433-1440.

61. Jones SM, Harris CPD, Lloyd J et al. Lipoproteins and their subfractions in psoriatic arthritis: Identification of an atherogenic profile with active joint disease. Ann Rheum Dis 2000; 59: 904-909.

62. Oliviero F, Nigro A Lo, Bernardi D et al. A comparative study of serum and synovial fl uid lipoprotein levels in patients with various arthritides. Clin Chim Acta 2012; 413: 303-307.

63. Uyanik BS, Ari Z, Onur E et al. Serum lipids and apolipoproteins in patients with psoriasis. Clin Chem Lab Med 2002; 40: 65-68.

Received September 13, 2018. Accepted October 19, 2018. 